\title{
Performance Analysis of Interweave Cognitive Radio Systems with Imperfect Channel Knowledge over Nakagami Fading Channels
}

\author{
Ankit Kaushik $^{*}$, Shree Krishna Sharma ${ }^{\dagger}$, Symeon Chatzinotas ${ }^{\dagger}$, Björn Ottersten ${ }^{\dagger}$, Friedrich Jondral ${ }^{*}$ \\ ${ }^{*}$ Communications Engineering Lab, Karlsruhe Institute of Technology (KIT), Germany \\ \{ankit.kaushik, friedrich.jondral\}@kit.edu \\ ${ }^{\dagger}$ SnT - securityandtrust.lu, University of Luxembourg, Luxembourg \\ \{shree.sharma, symeon.chatzinotas, bjorn.ottersten $\} @ u n i . l u$
}

\begin{abstract}
Knowledge of interacting channels is essential for characterizing the performance of a cognitive radio system in terms of interference power received by a primary receiver and throughput at a secondary receiver. Baseline models considered for the performance characterization assume perfect knowledge of the interacting channels. Recently, an analytical framework has been proposed that incorporates channel estimation and subsequently characterizes the performance of cognitive Interweave Systems (ISs). However, the analysis was pertained to the deterministic behaviour of the interacting channels. In this paper, we extend the characterization of the aforementioned framework to investigate the influence of channel fading on the performance of the IS. Our analysis indicate that an inappropriate choice of estimation time can severely degrade the performance of the IS in terms of achievable secondary throughput.
\end{abstract}

\section{INTRODUCTION}

The static allocation of the existing spectrum is largely responsible for causing scarcity in the spectrum. Cognitive Radio (CR) communication is foreseen as one of the potential contenders that could resolve this scarcity by utilizing the allocated spectrum efficiently. The widely investigated CR paradigms can be classified as follows: interweave, underlay and overlay systems [1]. Among these, Interweave Systems (ISs) are mostly preferred for theoretical analysis as well as for hardware implementations [2], [3]. The ISs depend on spectrum sensing to detect the presence of Primary User (PU) signals. Several techniques such as energy detection, matched filtering, cyclostationary and feature detection exist for detecting the PU signal [4]. Due to low complexity and versatility towards unknown PU signals, energy detection has been extensively employed for characterizing the performance of the IS [5]-[8]. In this regard, this paper focuses on the performance analysis of the cognitive ISs that employ estimation of the interacting channels, where the channels are subject to channel fading.

\section{A. Motivation and Related Work}

The performance of the IS can be characterized jointly in terms of interference (power) received at the Primary Receiver (PR) from the Secondary Transmitter (ST) and throughput achieved at the Secondary Receiver (SR). The interference at the PR depends on the detector's performance (depicted in terms of detection probability) employed at the ST. On the other side, false alarm probability largely contributes to the secondary throughput. Due to the employment of periodic sensing at the ST, the achievable secondary throughput is related to the sensing time. By operating at a desired detection probability, the interference at the PR can be regulated below a tolerance limit. Along with the secondary throughput, the false alarm and the detection probabilities depend on the sensing time. This relationship between the sensing time and the secondary throughput subject to a target detection probability has been investigated by Liang et. al. [6] as a sensingthroughput tradeoff. More specifically, the sensing-throughput tradeoff can be utilized for determining a suitable sensing time at which the maximum secondary throughput is achieved by the IS

It is worth noticing the fact that the detector employed for carrying out sensing is sensitive to the variations that arise due to presence of thermal noise in the system and fading in the channel. In this context, the characterization of the detection probability that captures the aforementioned variations has been carried out in the literature [9]-[11] Furthermore, Cardenas-Juarez et. al. [12] investigated the performance of the IS in terms of a sensing-throughput tradeoff. However, it is important to note that the performance analysis according to [10]-[12] assumes the perfect knowledge of the following interacting channels: sensing channel for the link PT-ST, access channel for the link ST-SR and interference channel for the link PT-ST, refer to Fig. 1. From a deployment perspective, this knowledge is not available at the ST. In this context, an analytical framework that employs channel estimation at the secondary system has been recently proposed in [13]. However, the performance analysis was confined to the deterministic behavior of the interacting channels. In this paper, we extend the performance analysis to study the effect of channel fading on the performance of the IS.

\section{B. Contributions}

In this paper, we provide the following contributions:

1) Analytical framework: We complement the analytical framework proposed in [13] by considering a random behaviour of the interacting channels (or channel fading). Based 


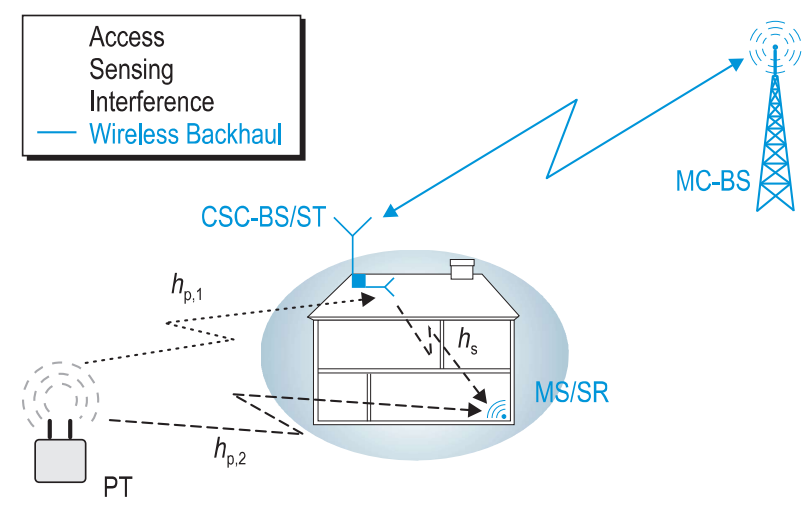

Fig. 1. A cognitive small cell scenario demonstrating: (i) the interweave paradigm, (ii) the associated network elements, which constitute cognitive small cell-base station/secondary transmitter (CSC-BS/ST), mobile station/secondary receiver (MS/SR), macro cell-base station (MC-BS) and primary transmitter (PT), (iii) the interacting channels: sensing channel $\left(h_{\mathrm{p}, 1}\right)$, access channel $\left(h_{\mathrm{s}}\right)$ and interference channel $\left(h_{\mathrm{p}, 2}\right)$.

on the expressions derived in this paper, we evaluate the performance of the IS that employs channel estimation, where the interacting channels are subject to Nakagami- $m$ fading. Nakagami- $m$ fading model, being a generalized fading model, facilitates in understanding the performance behaviour of ISs under different fading scenarios.

2) Estimation-sensing-throughput tradeoff: In order to capture the variations due to the channel estimation and the channel fading, we employ an outage constraint on the detection probability. Subsequently, we obtain an expression of the sensing-throughput tradeoff subject to the aforementioned constraint. We further exploit the tradeoff between the estimation time, the sensing time and the secondary throughput to determine a suitable estimation time and a suitable sensing time. In this regard, we adapt the estimation-sensingthroughput tradeoff proposed in [13] to the scenarios with channel fading.

\section{SYSTEM MODEL}

\section{A. Deployment scenario and Medium access}

The Cognitive Small Cell (CSC), a CR application, characterizes a small cell deployment that fulfills the spectral requirements for Mobile Stations (MSs) operating indoor, refer to Fig. 1. For the disposition of the CSC in the network, the following key elements are essential: a CSC-Base Station (CSC-BS), a Macro Cell-Base Station (MC-BS) and MS, refer to Fig. 1. Considering the fact that the IS is employed at the CSC-BS, the CSC-BS and the MS represent a ST and a SR, respectively.

Complementing the analysis depicted in [6], a slotted medium access for the IS is considered, according to which, the time axis is segmented into frames of length $T$. In order to incorporate channel estimation inside the frame, a frame structure that constitutes an estimation $\tau_{\text {est }}$, a sensing $\tau_{\text {sen }}$ and data transmission $T-\tau_{\text {sen }}$ is employed, where $\tau_{\text {est }}$ and $\tau_{\text {sen }}$ correspond to time intervals and $0<\tau_{\text {est }} \leq \tau_{\text {sen }}<T$, refer to Fig. 2 [13]. From a deployment perspective, the estimated values of the interacting channels are required for determining

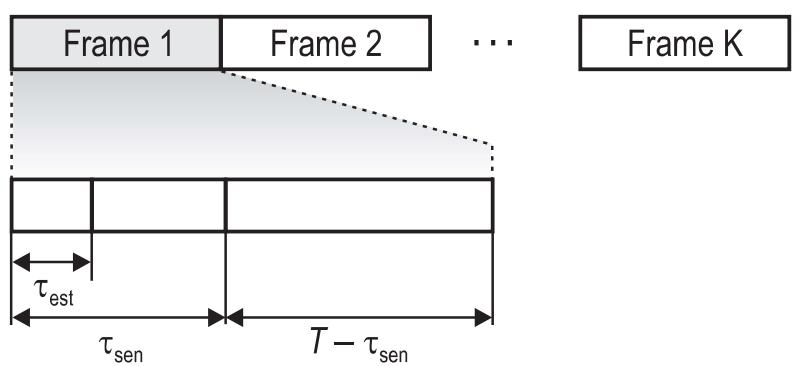

Fig. 2. An illustration of the frame structure for an interweave system depicting the estimation phase and the sensing phase for the sensing channel.

the suitable sensing time (the duration of the sensing phase). In this regard, the sequence (estimation followed by sensing) depicted in Fig. 2 is followed. As shown in the frame structure in Fig. 2, the samples (particularly for the sensing channel) used for estimation can be used for sensing such that the time resources within the frame duration can be utilized efficiently. It is important to note that the estimates for the interference and access channels at the ST are acquired by means of a low-rate feedback channel from the SR to the ST.

\section{B. Signal model}

Based on the underlying hypothesis that depicts the presence $\left(\mathcal{H}_{1}\right)$ or absence $\left(\mathcal{H}_{0}\right)$ of a PU signal, the discrete and real signal received at the $\mathrm{ST}$ is given by

$$
y_{\mathrm{ST}}[n]= \begin{cases}h_{\mathrm{p}, 1} \cdot x_{\mathrm{PT}}[n]+w[n] & : \mathcal{H}_{1} \\ w[n] & : \mathcal{H}_{0}\end{cases}
$$

where $x_{\mathrm{PT}}[n]$ corresponds to a discrete and real sample transmitted by the PT, $\left|h_{\mathrm{p}, 1}\right|^{2}$ represents the power gain of the sensing channel for a given frame and $w[n]$ is additive white Gaussian noise at the ST. According to [6], the signal $x_{\mathrm{PT}}[n]$ transmitted by the PUs can be modelled as: (i) a phase shift keying modulated signal, or (ii) a Gaussian signal. In this paper, we focus our analysis on the latter case. As a result, the mean and the variance for the signal and the noise are determined as $\mathbb{E}\left[x_{\mathrm{PT}}[n]\right]=0, \mathbb{E}[w[n]]=0$, $\mathbb{E}\left[\left|x_{\mathrm{PT}}[n]\right|^{2}\right]=\sigma_{x}^{2}$ and $\mathbb{E}\left[|w[n]|^{2}\right]=\sigma_{w}^{2}$. The channel $h_{\mathrm{p}, 1}$ is considered to be independent of $x_{\mathrm{PT}}[n]$ and $w[n]$, thus, $y_{\mathrm{ST}}$ is also an independent and identically distributed (i.i.d.) random process.

Similar to (1), during data transmission, the discrete and real received signal at the SR conditioned on the detection probability $\left(\mathrm{P}_{\mathrm{d}}\right)$ and false alarm probability $\left(\mathrm{P}_{\mathrm{fa}}\right)$ is given by

$$
y_{\mathrm{SR}}[n]=\left\{\begin{array}{ll}
h_{\mathrm{s}} \cdot x_{\mathrm{ST}}[n]+h_{\mathrm{p}, 2} \cdot x_{\mathrm{PT}}[n]+w[n] & : 1-\mathbf{P}_{\mathrm{d}} \\
h_{\mathrm{s}} \cdot x_{\mathrm{ST}}[n]+w[n] & : 1-\mathbf{P}_{\mathrm{fa}}
\end{array},\right.
$$

where $x_{\mathrm{ST}}[n]$ corresponds to discrete and real sample transmitted by the ST. Further, $\left|h_{\mathrm{s}}\right|^{2}$ and $\left|h_{\mathrm{p}, 2}\right|^{2}$ represent the power gains for the access and the interference channels, refer to Fig. 1.

\section{Channel fading}

Here, we characterize the channel gains $\left|h_{\mathrm{p}, 1}\right|^{2},\left|h_{\mathrm{p}, 2}\right|^{2}$ and $\left|h_{\mathrm{s}}\right|^{2}$ according to Nakagami- $m$ fading model. As a consequence, the power gains $\left|h_{\mathrm{p}, 1}\right|^{2},\left|h_{\mathrm{p}, 2}\right|^{2}$ and $\left|h_{\mathrm{s}}\right|^{2}$ follow a 
Gamma distribution [14], whose corresponding cumulative distribution functions are defined as

$$
\begin{array}{r}
F_{\left|h_{\mathrm{p}, 1}\right|^{2}}(x)=1-\Gamma\left(m_{\mathrm{p}, 1}, \frac{m_{\mathrm{p}, 1} x}{\left|h_{\mathrm{p}, 1}\right|^{2}}\right), \\
F_{\left|h_{\mathrm{p}, 2}\right|^{2}}(x)=1-\Gamma\left(m_{\mathrm{p}, 2}, \frac{m_{\mathrm{p}, 2} x}{\left|h_{\mathrm{p}, 2}\right|^{2}}\right), \\
F_{\left|h_{\mathrm{s}}\right|^{2}}(x)=1-\Gamma\left(m_{\mathrm{s}}, \frac{m_{\mathrm{s}} x}{\left|h_{\mathrm{s}}\right|^{2}}\right),
\end{array}
$$

where $m_{\mathrm{p}, 1}, m_{\mathrm{p}, 2}$ and $m_{\mathrm{s}}$ represent the Nakagami- $m$ parameter for $\left|h_{\mathrm{p}, 1}\right|^{2},\left|h_{\mathrm{p}, 2}\right|^{2}$ and $\left|h_{\mathrm{s}}\right|^{2}$, respectively, and $\Gamma(\cdot, \cdot)$ is a regularized upper-incomplete Gamma function [15].

\section{Theoretical Analysis}

\section{A. Perfect Channel Knowledge (Conventional Approach)}

We first consider a scenario (also represented as ideal model) that precludes channel estimation, in other words, the ST assumes the perfect knowledge of the interacting channels. In this context, the ST encounters variations caused due to the channel fading only. These variations translate to the variations in the detection probability, more specifically those variations that do not meet the desired detection probability $\left(\overline{\mathrm{P}}_{\mathrm{d}}\right)$ results in harmful interference at the PR. To overcome this issue, [12] proposed to employ an outage constraint over $P_{d}$, given as

$$
\mathbb{P}\left(\mathrm{P}_{\mathrm{d}} \leq \overline{\mathrm{P}}_{\mathrm{d}}\right) \leq \kappa,
$$

where $\kappa$ represents the outage constraint. Using (6), the ST is able to regulate harmful interference at the PR. As a result, a decision threshold $(\mu)$ on the $P_{\mathrm{Rx}, \mathrm{ST}}$ is obtained such that it satisfies the constraint defined (6) for a certain value of $\tau_{\text {sen }}$.

Besides the interference at the PR, the throughput at the $S R$ is given by (7), see top of the next page, where $C_{0}$ and $\mathrm{C}_{1}$ correspond to the data rate at the SR with and without interference from the PT. The signal to noise ratio for the links PT-ST and ST-SR are defined as: $\gamma_{\mathrm{p}, 1}=\frac{P_{\mathrm{R} x, S \mathrm{~T}}}{\sigma_{w}^{2}}-1$ and $\gamma_{\mathrm{s}}=\frac{\left|h_{\mathrm{s}}\right|^{2} P_{\mathrm{TX}, \mathrm{ST}}}{\sigma_{w}^{2}}$, respectively, and the interference to noise ratio for the link PT-SR is given by $\gamma_{\mathrm{p}, 2}=\frac{P_{\mathrm{R}, \mathrm{SR}}}{\sigma_{w}^{2}}-1$. Since the detection probability and the secondary throughput are related through the sensing time, this relationship is exploited to determine a sensing-throughput tradeoff for the case with the perfect channel estimation.

Theorem 1: Subject to an outage constraint on $\mathrm{P}_{\mathrm{d}}$, the sensing-throughput tradeoff that considers perfect channel estimation and random behaviour of the interacting channels, is given by

$$
\begin{aligned}
& R_{\mathrm{s}}\left(\tilde{\tau}_{\mathrm{est}}, \tilde{\tau}_{\mathrm{sen}}\right)=\max _{\tau_{\text {est }}, \tau_{\mathrm{sen}}} \mathbb{E}_{\mathrm{P}_{\mathrm{d}},\left|h_{\mathrm{s}}\right|^{2},\left|h_{\mathrm{p}, 2}\right|^{2}}\left[R_{\mathrm{s}}\left(\tau_{\mathrm{sen}}\right)\right], \\
& \text { s.t. }(6) \\
& \text { s.t. } 0<\tau_{\text {sen }} \leq T .
\end{aligned}
$$

Remark 1: It is worth noticing the fact the authors in [12] applied channel fading only to the sensing channel. However, according to Theorem 1, we consider a more practical approach, whereby, we exercise channel fading also over the access and the interference channels. Since the perfect channel knowledge scenario is employed to benchmark the performance of those ISs that employ channel estimation (discussed later in Section III-B), we evaluate the parameters such as threshold (which is used for evaluating $P_{d}$ and $P_{f a}$ ) and the expected secondary throughput numerically ${ }^{1}$.

\section{B. Imperfect Channel Knowledge (Proposed Approach)}

Here, we consider the estimation of the interacting channels, where the interacting channels encounter channel fading. To employ channel estimation, an estimation time is allocated within the frame duration of the IS, cf. Fig. 2. With this, the IS incorporates variations in the performance parameters $\left(\mathrm{P}_{\mathrm{d}}\right.$ and $\left.R_{\mathrm{s}}\right)$ due to the channel estimation and the channel fading. In order to facilitate the hardware complexity and the versatility to unknown PU signals (as proposed by the energy detector) requirements at the secondary system, we propose to employ received power-based estimation $\left(\hat{P}_{\mathrm{Rx}, \mathrm{ST}}, \hat{P}_{\mathrm{Rx}, \mathrm{SR}}\right)$ for the sensing and the interference channels at the ST and the SR and the pilot-based estimation $\left|\hat{h}_{s}\right|^{2}$ for the access channel. The characterization of the estimated parameters $\hat{P}_{\mathrm{Rx}, \mathrm{ST}}$ and $\hat{P}_{\mathrm{Rx}, \mathrm{SR}}$ for the sensing and the interference channel, and $\left|\hat{h}_{\mathrm{s}}\right|^{2}$ for the access channel in terms of their cumulative distribution functions (cdfs), for the deterministic case, has been performed in [13, cf. Section III-B]. The estimated parameters are used to estimate the performance parameters $\hat{\mathrm{P}}_{\mathrm{d}}, \hat{\mathrm{C}}_{0}$ and $\hat{\mathrm{C}}_{1}$, whose cdfs $F_{\hat{\mathrm{P}}_{\mathrm{d}}}, F_{\hat{\mathrm{C}}_{0}}$ and $F_{\hat{\mathrm{C}}_{1}}$ are characterized in [13, cf. Lemma 1, Lemma 2 and Lemma 3].

In order to protect the PR against harmful interference, we employ an outage constraint that jointly captures the variations due to the channel estimation and the channel fading, defined as

$$
\underbrace{\mathbb{E}_{\left|h_{\mathrm{p}, 1}\right|^{2}} \overbrace{\left[\mathbb{P}\left(\hat{\mathrm{P}}_{\mathrm{d}} \leq \overline{\mathrm{P}}_{\mathrm{d}}\right)\right]}^{\text {Channel Estimation }}}_{\text {Channel fading }} \leq \kappa,
$$

where $\mathbb{E}_{\left|h_{\mathrm{p}, 1}\right|^{2}}[\cdot]$ represents the expectation over the sensing channel. The variations due to the channel estimation only $\left(\mathbb{P}\left(\hat{\mathrm{P}}_{\mathrm{d}} \leq \overline{\mathrm{P}}_{\mathrm{d}}\right)\right)$ are characterized in terms of cdf as [13]

$$
F_{\hat{\mathrm{P}}_{\mathrm{d}}}(x)=1-\Gamma\left(\frac{\tau_{\mathrm{sen}} f_{\mathrm{s}}}{2}, \frac{\tau_{\mathrm{sen}} f_{\mathrm{s}}}{4 P_{\mathrm{Rx}, \mathrm{ST}} \Gamma^{-1}\left(x, \frac{\tau_{\mathrm{sen}} f_{\mathrm{s}}}{2}\right)}\right),
$$

where $\Gamma^{-1}(\cdot)$ represents the inverse function of regularized upper incomplete Gamma function. It is worth noticing that $\mathbb{E}_{\left|h_{\mathrm{p}, 1}\right|^{2}}[\cdot]$ in (9) acts on $P_{\mathrm{Rx}, \mathrm{ST}}$, as $P_{\mathrm{Rx}, \mathrm{ST}}$ incorporates the variations due to fading in the sensing channel $\left|h_{\mathrm{p}, 1}\right|^{2}$.

Next, we characterize the expression of the secondary throughput

$$
\begin{aligned}
R_{\mathrm{s}}\left(\tau_{\mathrm{est}}, \tau_{\mathrm{sen}}\right) & =\mathbb{E}_{\hat{\mathrm{P}}_{\mathrm{d}}, \hat{\mathrm{C}}_{0}, \hat{\mathrm{C}}_{1},\left|h_{\mathrm{p}, 1}\right|^{2},\left|h_{\mathrm{s}}\right|^{2},\left|h_{\mathrm{p}, 2}\right|^{2}}\left[\frac{T-\tau_{\mathrm{sen}}}{T} \times\right. \\
& \left.\left(\mathbb{P}\left(\mathcal{H}_{0}\right)\left(1-\mathrm{P}_{\mathrm{fa}}\right) \hat{\mathrm{C}}_{0}+\mathbb{P}\left(\mathcal{H}_{1}\right)\left(1-\hat{\mathrm{P}}_{\mathrm{d}}\right) \hat{\mathrm{C}}_{1}\right)\right]
\end{aligned}
$$

\footnotetext{
${ }^{1}$ In our future work, we plan to obtain analytical expressions of the aforementioned parameters.
} 


$$
R_{\mathrm{s}}(\tau)=\frac{T-\tau_{\mathrm{sen}}}{T} \mathbb{E}_{\left|h_{\mathrm{s}}\right|^{2}\left|h_{\mathrm{p}, 2}\right|^{2}}[\mathbb{P}\left(\mathcal{H}_{0}\right)\left(1-\mathrm{P}_{\mathrm{fa}}\right) \overbrace{\log _{2}\left(1+\frac{\left|h_{\mathrm{s}}\right|^{2} P_{\mathrm{Tx}, \mathrm{ST}}}{\sigma_{w}^{2}}\right)}^{\mathrm{C}_{0}}+\mathbb{P}\left(\mathcal{H}_{1}\right)\left(1-\mathrm{P}_{\mathrm{d}}\right) \overbrace{\log _{2}\left(1+\frac{\left|h_{\mathrm{s}}\right|^{2} P_{\mathrm{Tx}, \mathrm{ST}}}{\left|h_{\mathrm{p}, 2}\right|^{2} P_{\mathrm{Tx}, \mathrm{PT}}+\sigma_{w}^{2}}\right)}^{\mathrm{C}_{1}}]
$$

where $\mathbb{E}_{\hat{\mathrm{P}}_{\mathrm{d}}, \hat{\mathrm{C}}_{0}, \hat{\mathrm{C}}_{1},\left|h_{\mathrm{p}, 1}\right|^{2},\left|h_{\mathrm{s}}\right|^{2},\left|h_{\mathrm{p}, 2}\right|^{2}}[\cdot]$ corresponds to an expectation over the estimated parameters $\left(\hat{\mathrm{P}}_{\mathrm{d}}, \hat{\mathrm{C}}_{0}, \hat{\mathrm{C}}_{1}\right)$ and the channel fading $\left(\left|h_{\mathrm{p}, 1}\right|^{2},\left|h_{\mathrm{s}}\right|^{2},\left|h_{\mathrm{p}, 2}\right|^{2}\right)$. Please note that the random behaviour of the interacting channels due to the fading is included in the estimated system parameters $\hat{\mathrm{P}}_{\mathrm{d}}, \hat{\mathrm{C}}_{0}$ and $\hat{\mathrm{C}}_{1}$, refer to (7).

Theorem 2: Subject to an outage constraint on $\hat{\mathrm{P}}_{\mathrm{d}}$, the sensing-throughput tradeoff for the IS that considers imperfect channel estimation and random behaviour of the interacting channels, is given by

$$
\begin{aligned}
R_{\mathrm{s}}\left(\tilde{\tau}_{\text {est }}, \tilde{\tau}_{\text {sen }}\right) & =\max _{\tau_{\text {est }}, \tau_{\text {sen }}} \mathbb{E}_{\hat{\mathrm{P}}_{\mathrm{d}}, \hat{\mathrm{C}}_{0}, \hat{\mathrm{C}}_{1},\left|h_{\mathrm{p}, 1}\right|^{2},\left|h_{\mathrm{s}}\right|^{2},\left|h_{\mathrm{p}, 2}\right|^{2}}\left[R_{\mathrm{s}}\left(\tau_{\mathrm{est}}, \tau_{\text {sen }}\right)\right], \\
\text { s.t. } & (9), \\
\text { s.t. } & 0<\tau_{\text {est }} \leq \tau_{\text {sen }} \leq T .
\end{aligned}
$$

Proof: In order to solve the constrained optimization problem, the following approach is considered. We first exploit the underlying constraint (9) to determine the decision threshold $\mu$. Since it is complicated to obtain a closed form expression of $\mu$, in this regard, we obtain its value numerically.

Using $\mu$ to determine $\mathbb{E}_{\hat{\mathrm{P}}_{\mathrm{d}}}\left[\hat{\mathrm{P}}_{\mathrm{d}}\right]$ and $\mathrm{P}_{\mathrm{fa}}$ and evaluating an expectation over $\hat{\mathrm{P}}_{\mathrm{d}}, \hat{\mathrm{C}}_{0}, \hat{\mathrm{C}}_{1},\left|h_{\mathrm{p}, 1}\right|^{2},\left|h_{\mathrm{s}}\right|^{2},\left|h_{\mathrm{p}, 2}\right|^{2}$, we determine the expected secondary throughput as function of estimation and sensing time. Finally, this function is used to determine the suitable estimation time $\left(\tilde{\tau}_{\text {est }}\right)$ and sensing time $\left(\tilde{\tau}_{\text {sen }}\right)$.

In contrast to the ideal model (refer to Theorem 1), the sensing-throughput tradeoff investigated by the proposed approach (refer to Theorem 2) incorporates the imperfect channel knowledge. In this context, the performance characterization considered by the proposed framework is closer to the realistic situations.

Remark 2: Based on the expression $R_{\mathrm{s}}\left(\tau_{\mathrm{est}}, \tau_{\mathrm{sen}}\right)$ computed by the estimation model (referred as the proposed approach), we establish a fundamental relation between estimation time, sensing time and achievable throughput, this relationship is characterized as estimation-sensing-throughput tradeoff. Based on this tradeoff, we determine a suitable estimation $\tau_{\text {est }}=\tilde{\tau}_{\text {est }}$ and a sensing time $\tau_{\text {sen }}=\tilde{\tau}_{\text {sen }}$ that attains a maximum achievable throughput $R_{\mathrm{s}}\left(\tilde{\tau}_{\text {est }}, \tilde{\tau}_{\text {sen }}\right)$ for the IS.

\section{Numerical Results}

Here, we analyze the performance of the IS based on the proposed approach. To accomplish this: (i) we perform simulations to validate the expressions obtained in (9) and (11), (ii) we consider the ideal model to benchmark and evaluate the performance loss. Although the expressions derived using our sensing-throughput analysis are general and applicable to all CR systems, the parameters are selected in such a way that they closely relate to the deployment scenario described in Fig. 1. Unless stated explicitly, the choice of the parameters

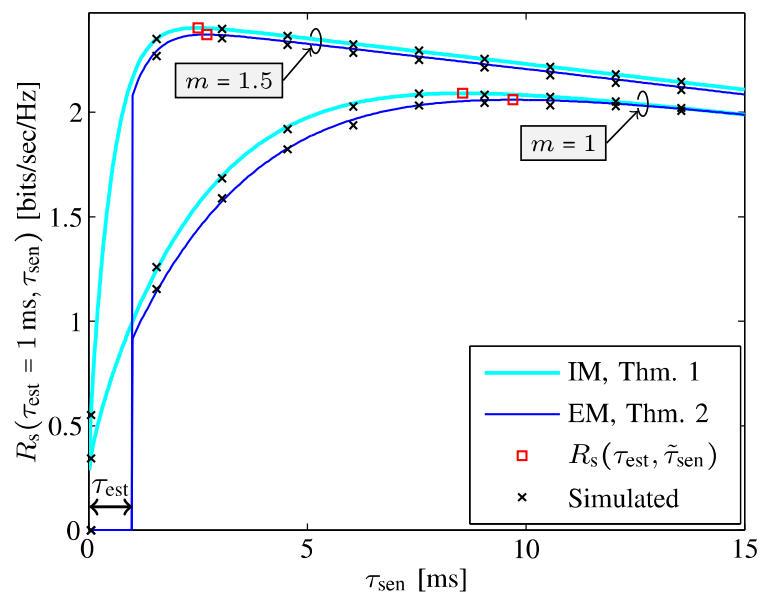

Fig. 3. Sensing-throughput tradeoff for the ideal model (IM) and estimation model $(\mathrm{EM}), \gamma_{\mathrm{p}, 1}=0 \mathrm{~dB}, \tau_{\mathrm{est}}=1 \mathrm{~ms}$ and $\kappa=0.05$.

$f_{\mathrm{s}}=1 \mathrm{MHz}, h_{\mathrm{p}, 1}, h_{\mathrm{p}, 2}=-100 \mathrm{~dB}, h_{\mathrm{s}}=-80 \mathrm{~dB}, T=100 \mathrm{~ms}$, $\overline{\mathrm{P}}_{\mathrm{d}}=0.9, \kappa=0.05, \sigma_{w}^{2}=-100 \mathrm{dBm}, \gamma_{\mathrm{p}, 1}=0 \mathrm{~dB}, \gamma_{\mathrm{p}, 2}=0 \mathrm{~dB}$, $\gamma_{\mathrm{s}}=10 \mathrm{~dB}, \sigma_{x}^{2}=P_{\mathrm{Tx}, \mathrm{PT}}=0 \mathrm{dBm}, P_{\mathrm{Tx}, \mathrm{ST}}=-10 \mathrm{dBm}$, $\mathbb{P}\left(\mathcal{H}_{1}\right)=1-\mathbb{P}\left(\mathcal{H}_{0}\right)=0.2, \tau_{\text {est }}=1 \mathrm{~ms}$ and $N_{\mathrm{s}}=10$ is considered for the analysis. In addition, we investigate the performance of the IS under the following fading scenarios : (i) severe fading $m=1$ (Rayleigh fading), and (ii) mild fading $m=1.5$.

First, we investigate the sensing-throughput tradeoff for a certain value of estimation time $\tau_{\text {est }}=1 \mathrm{~ms}$, corresponding to the Ideal Model (IM) and Estimation Model (EM) that represent the perfect and the imperfect channel estimation, respectively, refer to Fig. 3. It is observed that with the inclusion of $\tau_{\text {est }}$ in the frame structure, the EM procures no throughput at the SR for the time interval $\tau_{\text {est }}$. Furthermore, it is noticed that the suitable sensing time increases with the severity in the fading. To procure further insights, we consider the variation of other parameters on the performance of the IS.

Upon maximizing the secondary throughput for a certain $\tau_{\text {est }}$, we consider the variation of $R_{\mathrm{s}}\left(\tau_{\text {est }}, \tilde{\tau}_{\text {sen }}\right)$ along the estimation time, refer to Fig. 4 . It is noticed that $R_{\mathrm{s}}\left(\tau_{\text {est }}, \tilde{\tau}_{\text {sen }}\right)$ increases for low values of $\tau_{\text {est }}$ and then decreases beyond $\tilde{\tau}_{\text {est }}$. This can explained as follows, low $\tau_{\text {est }}$ increases the variations in $\hat{P}_{d}$, shifting the threshold to lower values, which subsequently increases $\mathrm{P}_{\mathrm{fa}}$, hence, degrading the achievable secondary throughput. Beyond $\tilde{\tau}_{\text {est }}$, the variations are largely dominated by the channel fading, therefore, the IS observes no improvement by increasing $\tau_{\text {est }}$. Moreover, it is observed that the mild fading scenarios are more sensitive to the performance degradation in terms of the secondary throughput. Fig. 5 considers the variation of expected detection probability against $\tau_{\text {est }}$. It is observed that, despite the variations due to the channel estimation and the channel fading considered by the EM, the outage constraint is satisfied for all values of $\tau_{\text {est }}$. 


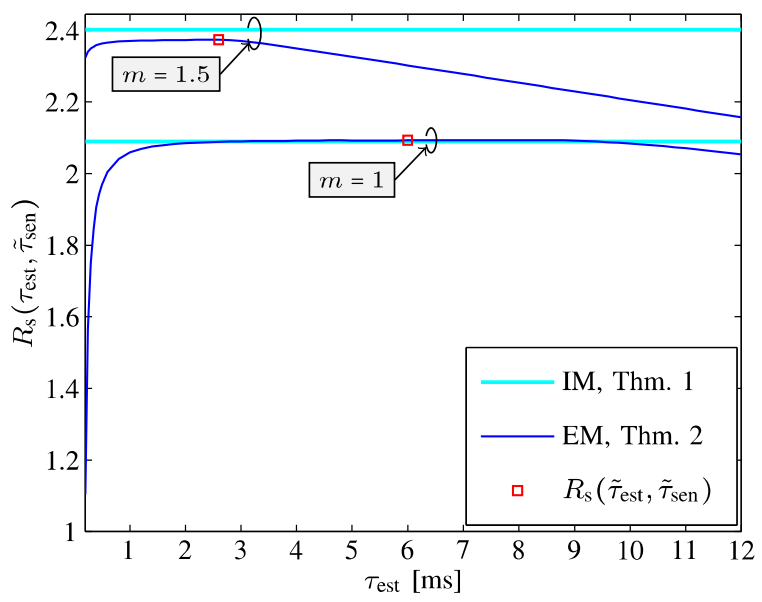

Fig. 4. Estimation-sensing-throughput tradeoff for the outage constraint with $\gamma_{\mathrm{p}, 1}=0 \mathrm{~dB}$, where the secondary throughput is maximized over the sensing time, $R_{\mathrm{s}}\left(\tau_{\text {est }}, \tilde{\tau}_{\text {sen }}\right)$. Estimation-sensing-throughput tradeoff is utilized to determine a suitable estimation time $\tilde{\tau}_{\text {est }}$ that maximizes the secondary throughput, $R_{\mathrm{s}}\left(\tilde{\tau}_{\text {est }}, \tilde{\tau}_{\text {sen }}\right)$.

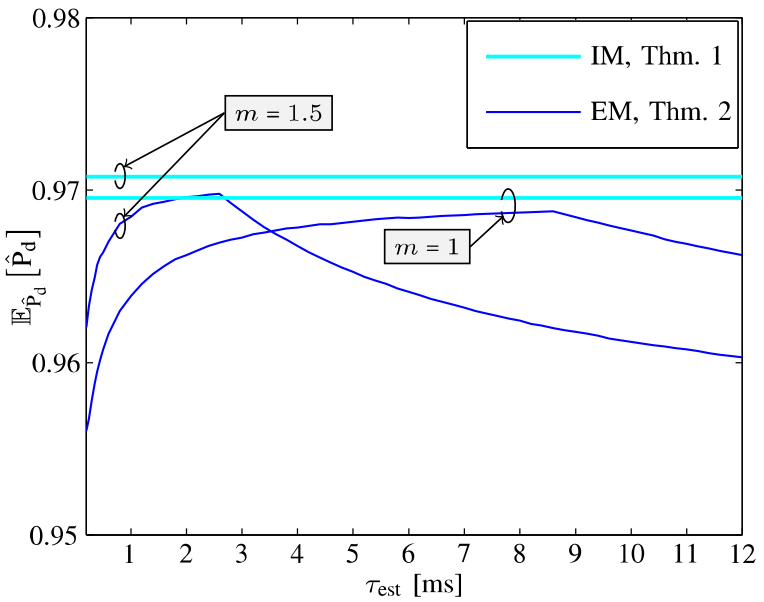

Fig. 5. Expected detection probability versus estimation time.

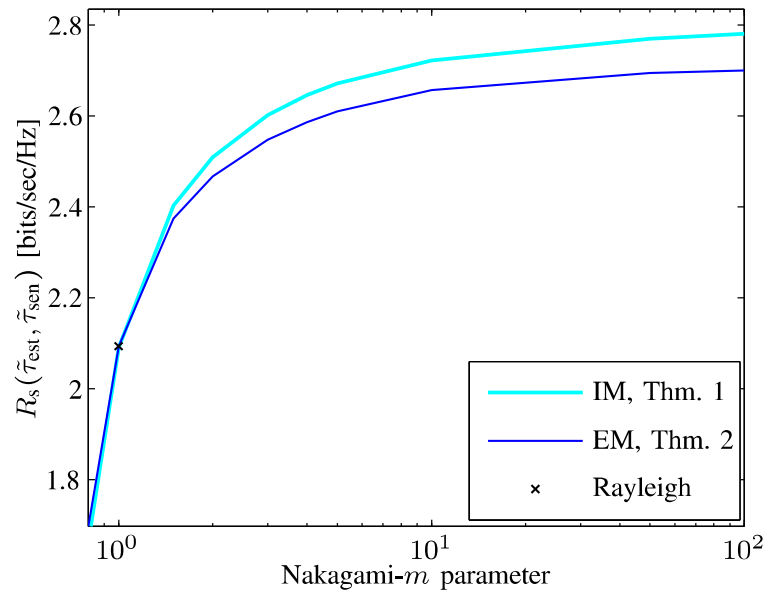

Fig. 6. Variation of the achievable throughput $R\left(\tilde{\tau}_{\text {est }}, \tilde{\tau}_{\text {sen }}\right)$ with Nakagami$m$ parameter for $\gamma_{\mathrm{p}, 1}=0 \mathrm{~dB}$.

Finally, we investigate the performance degradation in terms of achievable secondary throughput $R_{\mathrm{s}}\left(\tilde{\tau}_{\text {est }}, \tau_{\text {sen }}\right)$ versus the Nakagami- $m$ parameter that accounts for severity in the fading, refer to Fig. 6. While comparing the IM and the EM, it can be concluded that, for situations where the variations in the system are largely dominated by the channel estimation, a greater performance degradation is observed by the EM.

\section{CONCLUSION}

In this paper, we characterized the performance of the interweave systems that incorporate imperfect knowledge of the interacting channels, considerding these channels are subject to Nakagam- $m$ fading. An outage constraint that jointly captures the variations in the IS due to channel estimation and channel fading has been employed. Subject to this constraint, a sensing-throughput tradeoff that incorporates channel estimation and channel fading has been characterized that yields a maximum secondary throughput at a suitable estimation and a sensing time. Finally, it has been concluded that the suitable choice of the estimation time is essential for controlling the performance degradation in terms of the achievable secondary throughput, particularly for scenarios that encounter less severe (mild) fading.

\section{REFERENCES}

[1] A. Goldsmith, S. Jafar, I. Maric, and S. Srinivasa, "Breaking Spectrum Gridlock With Cognitive Radios: An Information Theoretic Perspective," Proceedings of the IEEE, vol. 97, no. 5, pp. 894-914, May 2009.

[2] A. Kaushik, M. Mueller, and F. K. Jondral, "Cognitive Relay: Detecting Spectrum Holes in a Dynamic Scenario," in ISWCS, Apr. 2013, pp. 1-2.

[3] A. Kaushik, F. Wunsch, A. Sagainov, N. Cuervo, J. Demel, S. Koslowski, H. Jakel, and F. Jondral, "Spectrum sharing for $5 \mathrm{G}$ wireless systems (Spectrum sharing challenge)," in IEEE International Symposium on Dynamic Spectrum Access Networks (DySPAN), Sept 2015, pp. 1-2.

[4] S. Sharma, T. Bogale, S. Chatzinotas, B. Ottersten, L. Le, and X. Wang, "Cognitive Radio Techniques under Practical Imperfections: A Survey," IEEE Communications Surveys Tutorials, vol. 17, no. 4, pp. 1858-1884, Fourthquarter 2015.

[5] R. Tandra and A. Sahai, "SNR Walls for Signal Detection," IEEE Journal of Selected Topics in Signal Processing, vol. 2, no. 1, pp. 4-17, Feb 2008.

[6] Y.-C. Liang, Y. Zeng, E. Peh, and A. T. Hoang, "Sensing-Throughput Tradeoff for Cognitive Radio Networks," IEEE Transactions on Wireless Communications, vol. 7, no. 4, pp. 1326-1337, April 2008.

[7] Y. Sharkasi, M. Ghogho, and D. McLernon, "Sensing-throughput tradeoff for OFDM-based cognitive radio under outage constraints," in ISWCS, Aug 2012, pp. 66-70.

[8] H. Pradhan, S. Kalamkar, and A. Banerjee, "Sensing-Throughput Tradeoff in Cognitive Radio With Random Arrivals and Departures of Multiple Primary Users," IEEE Communications Letters, vol. 19, no. 3, pp. 415418, March 2015

[9] V. Kostylev, "Energy detection of a signal with random amplitude," in IEEE ICC, vol. 3, 2002, pp. 1606-1610.

[10] F. Digham, M.-S. Alouini, and M. K. Simon, "On the energy detection of unknown signals over fading channels," in IEEE ICC, vol. 5, May 2003, pp. 3575-3579.

[11] S. Herath, N. Rajatheva, and C. Tellambura, "Unified Approach for Energy Detection of Unknown Deterministic Signal in Cognitive Radio Over Fading Channels," in IEEE ICC Workshops, June 2009, pp. 1-5.

[12] M. Cardenas-Juarez and M. Ghogho, "Spectrum Sensing and Throughput Trade-off in Cognitive Radio under Outage Constraints over Nakagami Fading," IEEE Communications Letters, vol. 15, no. 10, pp. 11101113, October 2011.

[13] A. Kaushik, S. K. Sharma, S. Chatzinotas, B. Ottersten, and F. K Jondral, "Sensing-Throughput Tradeoff for Cognitive Radio as Interweave System: A Deployment-Centric Viewpoint," IEEE Transactions on Wireless Communications, vol. PP, no. 99, pp. 1-1, 2016.

[14] A. Goldsmith, Wireless Communications. New York, NY, USA Cambridge University Press, 2005.

[15] M. Abramowitz and I. A. Stegun, Handbook of Mathematical Functions with Formulas, Graphs, and Mathematical Tables, ninth Dover printing, tenth GPO printing ed. New York: Dover, 1964 